\title{
Pandemia y discapacidad. Lecciones a propósito del confinamiento
}

\author{
Pandemic and disability. Lessons from confinement
}

\section{Palabras clave}

Discapacidad, pandemia, COVID-19, inclusión social, capacitismo.

\section{Keywords}

Disability, pandemic, COVID-19, social inclusion, ableism.

\section{Agustín Huete García <ahueteg@usal.es>}

INICO, Universidad de Salamanca. España

\section{Para empezar... una obviedad}

En medio de la tremenda crisis generada por la pandemia del COVID-19, parece lógico pensar que las personas con discapacidad, sus familias y las organizaciones que les prestan apoyo, han sufrido las consecuencias de manera extraordinaria y, efectivamente, mientras escribo estas líneas se suceden las evidencias sobre ello. Aunque el momento es prematuro y el tiempo transcurrido breve, ya empiezan a aparecer trabajos que tratan de aquilatar estas consecuencias.

Tal como ha reconocido recientemente el Secretario General de Naciones Unidas, la pandemia de COVID-19 está intensificando las desigualdades experimentadas por millones de personas en el mundo, y también las personas con discapacidad (ONU, 6 de mayo de 2020), que están siendo víctimas de discriminación ya desde el momento de recibir tratamiento. Sin entrar en detalles, ya que no es el tema central de este texto, la propia Alta Comisionada de las Naciones Unidas para los Derechos Humanos, Michelle Bachelet, ha mostrado su preocupación sobre si la vida de las personas con discapacidad puede haber tenido un "peso diferente al de otros durante esta pandemia" (ONU, 30 de abril de 2020).

Citaré aquí algunos temas destacados en publicaciones o instituciones de referencia. En el terreno de la prevención, por ejemplo, atender algo tan

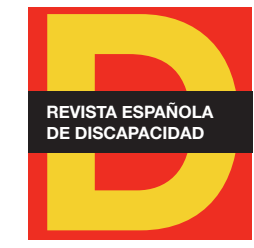

Para citar:

Huete, A. (2020). "Pandemia y discapacidad. Lecciones a propósito del confinamiento". Revista Española de Discapacidad, 8(I), pp. 203-207.

Doi: <https://doi.org/10.5569/23405104.08.01.11> 
simple como las dos medidas básicas para evitar el contagio (la higiene de manos y la distancia social) son en sí mismas una dificultad para personas con discapacidad con grandes necesidades de apoyo, obligadas a un contacto permanente para realizar cualquier actividad básica o instrumental de la vida diaria (Armitage y Nellmus, 2020).

Otro tema emergente ha sido el de la provisión de información de manera inclusiva. Por un lado, el más obvio, en relación con los protocolos, guías y otros elementos de información, afectados con frecuencia por problemas de accesibilidad para personas con discapacidades de la visión, audición o cognitivas. Por otro, en relación con la generación, acumulación y difusión de información estadística general sobre la pandemia, en los que de manera generalizada se ha obviado a la población con discapacidad¹.

Por último, resulta relevante, tal como ha mostrado la Fundación CERMI Mujeres en una guía publicada con ocasión de la crisis COVID-19, que la emergencia sanitaria conlleva un aumento del riesgo de violencia machista y sexual, con especial incidencia entre las mujeres con discapacidad (Fundación CERMI Mujeres, 12 de mayo de 2020).

Valgan estos cuatro ejemplos para mostrar que, efectivamente, las poblaciones afectadas por discriminación capacitista (Otaola y Huete, 2019), sufren también de manera extraordinaria en momentos de crisis como esta. Siguiendo a Spiney (12 de abril de 2020), las medidas diseñadas para frenar la propagación de la enfermedad y evitar el colapso de los sistemas de salud pueden, efectivamente, estar empeorando las desigualdades sociales.

\section{El encierro como experimento}

Escriben Stevens y Landes (2020) que la crisis del COVID-19 puede funcionar como "el canario en la mina de carbón", advirtiendo de la acumulación de vapores nocivos que, en estado normal, nuestra sociedad destila en pequeñas dosis, en cantidades soportables al fin y al cabo. Ni siquiera es preciso fijarse en todos los aspectos de la crisis, basta con centrarse en uno de ellos, el confinamiento, como muestra de que esto puede ser cierto, y por tanto, útil.

A lo largo y ancho del planeta, la mayoría de los gobiernos han decretado el encierro de la población en sus casas, y lo han hecho además concentrando la toma de decisiones, es decir, el poder, en unas pocas manos. Por ejemplo en España, mediante el decreto la declaración de Estado de Alarma y la centralización de las decisiones en unos pocos ministerios, fundamentalmente, el de Sanidad (Real Decreto 463/2020, de 14 de marzo).

\footnotetext{
1. A pesar de que el Comité Español de Representantes de Personas con Discapacidad (CERMI), con apoyo del Instituto Nacional de Estadística (INE), solicitó al Instituto de Salud Carlos III -siguiendo el mandato claro de Naciones Unidas- la inclusión de la discapacidad como variable a registrar en estudio de seroprevalencia, la medida no se llegó a implementar renunciando así a obtener información sobre el impacto del COVID-19 en una población reconocida como especialmente vulnerable.
} 
Las medidas de confinamiento han sido justificadas de manera manifiesta por la preservación de la capacidad del sistema sanitario para atender a las personas afectadas. Esta primacía de lo médico se ha impuesto sin dificultad en la vida diaria a través de órdenes ministeriales y medios de comunicación, lo que en suma ha supuesto evidenciar la preeminencia de lo puramente médico-biológico como criterio fundamental de organización social, cuestión que, seguramente, dará para análisis más detallados.

Si lo pensamos detenidamente, el encierro de la población ha supuesto, además, la eliminación de la vida pública presencial, tradicional, física. La ciudadanía se ha visto obligada, por razones bien justificadas claro que sí, a permanecer en la opacidad del hogar, del mundo privado, y a buscar resignadamente la presencia social, como mucho, desde los balcones (Calvo y Bejarano, 2020) y las redes sociales.

Igualmente, el confinamiento ha supuesto la pérdida grave de oportunidades laborales, pero no exactamente por falta de empleo o cualificación, o por una crisis financiera o de otro tipo que destruye la economía. De alguna manera se podría considerar como una renuncia forzada pero asumida de buen grado, aceptando que más importante incluso que tener trabajo, es cuidar a otros y evitar que sufran o lo peor, mueran.

Dicho de otra manera, el éxito del confinamiento ha dependido en buena parte del consentimiento, o incluso, se podría decir, del acomodamiento. De la aceptación de que, aun a pesar de las molestias, no sólo se puede sobrevivir encerrado en casa, sino que se puede llegar a estar bien, y se puede aprovechar para hacer cosas que elevan el espíritu, o al menos, lo entretienen. Todo ello, además, aceptando buenas dosis de incertidumbre en los plazos y procesos de resolución de la crisis.

En definitiva, el encierro ha supuesto la pérdida -impuesta pero consentida- de grandes cuotas de autonomía personal de millones de personas a la vez. Siguiendo la definición clásica introducida en la filosofía por Kant (Elton y Mauri, 2013) la autonomía es la situación en que el comportamiento o la voluntad del individuo está determinada por su propio entendimiento, y no por algo o alguien ajeno. De hecho, Kant presenta a la autonomía como fuente principal de dignidad de la naturaleza humana (Cabrera, 2002).

Los estudios sociales de la discapacidad han demostrado que la discriminación por discapacidad supone precisamente una pérdida generalizada de la autonomía personal, basada en: la primacía de lo médico sobre lo social, el aislamiento mediante barreras para el uso del espacio y el conocimiento público, la pérdida grave de oportunidades laborales independientemente de la cualificación o la pujanza de la economía, la pérdida de oportunidades para tomar decisiones sobre la propia vida y ello, en un contexto en el que todo ocurre por el bien de las propias personas con discapacidad y, en muchos casos, con su consentimiento más o menos forzoso.

Especialmente importante resulta esto de la aceptación o el consentimiento. Son muchos los investigadores sociales que han demostrado cómo es imprescindible la voluntad de las minorías étnicas para sostener el racismo, o la complicidad de las mujeres para sostener el machismo. Igualmente, en muchos estudios que hemos realizado con metodología cualitativa sobre personas con discapacidad hemos encontrado ese 'acomodamiento' de las propias personas con discapacidad y sus familias a una situación de desventaja impuesta, sine die en la mayoría de los casos. 


\section{La pandemia como oportunidad}

Dice el filósofo italiano Nuccio Ordine (Ayén, 3 de mayo de 2020) que esta pandemia es una ocasión única para revisar la vida social y, si es posible, aprovechar para tomar decisiones. El confinamiento es seguramente un experimento de encierro global nunca antes visto en la historia de la humanidad y como tal, como experimento, puede ser aprovechado.

Quienes por razones personales o profesionales conocemos la discriminación por discapacidad, a menudo sentimos frustración por lo difícil que resulta no ya su erradicación, siquiera su contención. Es abrumadora la frecuencia con que, una y otra vez, nos encontramos ejemplos de discriminación por discapacidad en la vida cotidiana, o en los contextos más insospechados. Frustración, que suele ser seguida por una constatación: es necesario explicar más y mejor la discriminación por discapacidad, sobre todo a las personas que ignoran totalmente su existencia.

Pareciera que, a pesar de que inagotables evidencias sostienen que las personas con discapacidad sufren en casi cualquier escenario un trato desigual, la sociedad se muestra inamoviblemente resistente al cambio. No es fácil provocar por tanto una transformación social en sentido positivo hacia la población con discapacidad, y conviene aprovechar cualquier oportunidad para intentarlo. ¿Es la pandemia COVID-19 una oportunidad para ello? ¿Se puede extraer algo positivo de todo este desastre?

La avezada lectora, el avezado lector, habrá caído ya en la cuenta a estas alturas de que el gran experimento del confinamiento puede haber servido para que millones de personas, a la vez, experimenten qué significa vivir en situación de discriminación por discapacidad. Cualquier forma de discriminación en realidad.

Lo que toca ahora, y cuanto antes mejor para que no se pierda la memoria, es intentar aprovechar la experiencia vivida por tantas personas, como ejemplo de lo que supone vivir con una desventaja impuesta y, generalmente, aceptada. No está claro que así sea, de hecho casi a la vez que escribo estas líneas, Víctor Villar en Infolibre (3 de mayo de 2020) se muestra poco optimista sobre la posibilidad de que este experimento del confinamiento pueda servir para provocar un gran ejercicio "de empatía de toda la población con las personas con discapacidad".

Ahora bien al menos, eso sí, podrá servir para que muchas personas sin discapacidad tomen nota y ejemplo de competencias básicas para la supervivencia en confinamiento que las personas con discapacidad tienen más que dominadas. Dicho de otra forma, para que, al menos por una vez, las personas con discapacidad puedan ser consideradas como valiosas integrantes de la sociedad, por su experiencia, conocimiento y habilidad para adaptarse y sobrevivir en tiempos de pandemia. 


\section{Referencias bibliográficas}

Armitage, R. y Nellmus, L. (2020). "The COVID-19 response must be disability inclusive". The Lancet Public Health, 5(5), pp. e257.

Ayén, X. (16 marzo, 2020). Nuccio Ordine: "El coronavirus nos muestra que las personas no son islas". La Vanguardia. Conversaciones de la Pandemia. Recuperado de https://cutt.ly/zyC6Mqs.

Cabrera, P. (2002). “Cárcel y exclusión”. Revista del Ministerio de Trabajo y Asuntos Sociales, 35, pp. 83-120.

Calvo, K. y Bejarano, E. (29 mayo, 2020). Music, solidarity and balconies in Spain. Interface: a journal for and about social movements. Recuperado de https://cutt.ly/tyC6Tel.

Elton, M. y Mauri, M. (2013). "La Heteronomía de la voluntad Kantiana. Una comparación con Tomás de Aquino". Revista Pensamiento, 69(258), pp. 115-129.

Fundación CERMI Mujeres (12 mayo, 2020). Cuidados en la COVID-19 desde la perspectiva de género y discapacidad. Recuperado de https://cutt.ly/YyC7Ekn.

ONU (6 mayo, 2020). Las personas con discapacidad no pueden ser aún más discriminadas a causa del coronavirus. Recuperado de https://cutt.ly/SyC85jp.

ONU (30 abril, 2020). Bachelet insta a los Estados a tomar medidas adicionales para incluir a las personas con discapacidad. Comunicados de la Alta Comisionada. Recuperado de https://cutt.ly/GyC83dL.

Otaola, M. y Huete, A. (2019). "Capacitismo: un fenómeno sociodemográfico". Actas de Coordinación Sociosanitaria, 25, pp. 179-198.

España. Real Decreto 463/2020, de 14 de marzo, por el que se declara el estado de alarma para la gestión de la situación de crisis sanitaria ocasionada por el COVID-19, Boletín Oficial del Estado, 14 de marzo de 2020, núm. 67, pp. 25390 a 25400. Recuperado de https://www.boe.es/eli/es/rd/2020/03/14/463.

Spiney, L. (12 abril, 2020). Historically, disease outbreaks have happened at a time of social inequality and discord. The Guardian. Recuperado de https://cutt.ly/GyC5nd4.

Stevens, D. y Landes, S. (2020). "Potential Impacts of COVID-19 on Individuals with Intellectual and Developmental Disability: A Call for Accurate Cause of Death Reporting". Lerner Center for Public Health Promotion. Research Brief, 20. Recuperado de https://cutt.ly/5yC5NwQ.

Villar, V. (3 mayo, 2020). Discapacidad y dependencia en tiempos de covid-19 (Parte I: Miedo). Infolibre. Recuperado de https://cutt.ly/9yVqiVZ. 